\title{
Extremal Problems for a Class of Symmetric Functions
}

\author{
Renate McLaughlin
}

Communicated by M. M. SCHIFFER

\section{Introduction}

Let $R$ denote the annulus $\left\{z: r_{0}<|z|<1\right\}$. D. Garer [3] introduced the family $\mathscr{F}$ of functions $f(z)$ that are holomorphic and schlicht in $R$ and satisfy the three conditions

$$
\begin{gathered}
|f(z)|<1 \quad(z \in R), \quad|f(z)|=1 \quad(|z|=1), \\
f(z) \neq 0 \quad(z \in R), \\
f(1)=1 .
\end{gathered}
$$

Since then, many extremal problems for the class $\mathscr{F}$ and related classes have been considered ([1], [2], [4] to [9]).

In many of these extremal problems, the extremal function is symmetric with respect to the real axis; or if an extremal function is not unique, there exists a symmetric extremal function [7]. This leads us to consider the compact subclass $\mathscr{F}_{s}$ of functions whose image is symmetric with respect to the real axis:

$$
\mathscr{F}_{s}=\{f: f \in \mathscr{F}, f(z)=\overline{f(\bar{z})}\} .
$$

We shall develop a variational formula for $\mathscr{F}_{s}$ and use it to solve extremal problems in $\mathscr{F}_{s}$. In most cases, we shall obtain unique extremal functions. Several extremal problems that appear to be inaccessible in $\mathscr{F}$ (because many parameters are involved) can easily be solved in the subclass $\mathscr{F}_{s}$.

Of course, if an extremal function for $\mathscr{F}$ belongs to $\mathscr{F}_{s}$, it is also extremal in the smaller class. Thus, in Sections 3 and 4, our method yields known results.

\section{A Variational Formula for $\mathscr{F}_{s}$}

Each $f \in \mathscr{F}$ maps $R$ onto the unit disk minus some continuum $\Gamma_{f}$ containing the origin. If $f \in \mathscr{F}_{s}$, then $\Gamma_{f}$ is symmetric with respect to the real axis.

Now let $f$ belong to $\mathscr{F}_{s}$. Fix $w_{0} \in \Gamma_{f}$, say $w_{0} \neq 0$. Let $D_{\rho}\left(w_{0}\right)(\rho>0)$ denote the domain consisting of all points either exterior to $\Gamma_{f}$ or exterior to the disk $\left|w-w_{0}\right| \leqq \rho$. It is known [2], [10] that there exist functions of the form

$$
F(w)=w+\frac{a \rho^{2} w}{\left(w-w_{0}\right) w_{0}}+O\left(\rho^{3}\right)
$$


that are analytic and univalent in $D_{\rho}\left(w_{0}\right)$ and that leave the origin fixed. Here the constant $a$ depends on $\rho$ and $|a|=|a(\rho)| \leqq 1$, and the error term $O\left(\rho^{3}\right)$ can be estimated uniformly in each closed subdomain of $D_{\rho}\left(w_{0}\right)$.

If $w_{0}$ is real, it follows [10] that the function $F(w)$ can be chosen to have real coefficients, so that $F(\bar{w})=\overline{F(w)}$. Now suppose $w_{0}$ is not real, say $\operatorname{Im} w_{0}>0$. Choose $\rho$ so small that $\left|w-w_{0}\right|=\rho$ does not intersect the real axis. There exists a function $h(w)=w /\left(w-\bar{w}_{0}\right)+O(\rho)$, defined and satisfying a Lipschitz condition in the half-plane $\operatorname{Im} w>\operatorname{Im} \bar{w}_{0} / 2$ (this implies that for each constant $c$ the function $w+c \rho^{2} h(w)$ is univalent for sufficiently small $\rho$ ), such that

$$
H(w)=F(w)+\frac{\bar{a} \rho^{2}}{\bar{w}_{0}} h(F(w))
$$

is univalent in $D_{\rho}\left(w_{0}\right) \cap\{w: \operatorname{Im} w \geqq 0\}$ and maps the real axis onto the real axis with $H(0)=0$, for all sufficiently small $\rho$. A computation shows that

$$
H(w)=w+\frac{a \rho^{2} w}{\left(w-w_{0}\right) w_{0}}+\frac{\bar{a} \rho^{2} w}{\left(w-\bar{w}_{0}\right) \bar{w}_{0}}+O\left(\rho^{3}\right) .
$$

Now extend $H$ to a univalent function in $D_{\rho}\left(w_{0}\right) \cap D_{\rho}\left(\bar{w}_{0}\right)$ by setting $H(\bar{w})=\overline{H(w)}$. (Clearly, if $w_{0}$ is real, then $a$ can be chosen to be real, and $H$ has the same form as $F$.)

Proceeding as in [2], computing first the function

$$
W-\frac{a \rho^{2} W^{2}}{\left(1-w_{0} W\right) w_{0}}-\frac{\bar{a} \rho^{2} W^{2}}{\left(1-\bar{w}_{0} W\right) \bar{w}_{0}}, \quad W=H(w),
$$

we find the following variational formula for the class $\mathscr{F}_{s}$ :

$V_{\rho}^{s}(w)=w\left[1+a \rho^{2} \frac{1-w^{2}}{w_{0}\left(w-w_{0}\right)\left(1-w_{0} w\right)}+\bar{a} \rho^{2} \frac{1-w^{2}}{\bar{w}_{0}\left(w-\bar{w}_{0}\right)\left(1-\bar{w}_{0} w\right)}\right]+O\left(\rho^{3}\right)$.

\section{Maximum and Minimum Value of $|f(z)|$}

Suppose that $f$ is an extremal function for the problem

$$
\max _{g \in \mathscr{F}_{s}}|g(z)| \text {. }
$$

Then $\left|V_{\rho}^{s}(f(z))\right| \leqq|f(z)|$. Set $\alpha=f(z)$. The last inequality leads to the relation

$$
\operatorname{Re}\left\{a \rho^{2}\left[\frac{1-\alpha^{2}}{w_{0}\left(\alpha-w_{0}\right)\left(1-\alpha w_{0}\right)}+\frac{1-\bar{\alpha}^{2}}{w_{0}\left(\bar{\alpha}-w_{0}\right)\left(1-\bar{\alpha} w_{0}\right)}\right]+O\left(\rho^{3}\right)\right\} \leqq 0 .
$$

It now follows from SCHIFFE's lemma [10] that the continuum $\Gamma_{f}$ satisfies the differential equation $w^{\prime}(t)^{2} s(w(t))>0$, where

$$
s(w)=\frac{\operatorname{Re} \alpha-w\left(1+|\alpha|^{2}\right)+w^{2} \operatorname{Re} \alpha}{w(\alpha-w)(1-\alpha w)(\bar{\alpha}-w)(1-\bar{\alpha} w)} .
$$


Case I. $\operatorname{Re} \alpha=0$. Because of the symmetry, it suffices to consider $\alpha=i \beta(\beta>0)$. The solution curve containing the origin is $w(t)=i \beta t(-1<t<1)$, and $\Gamma_{f}$ would be an interval [ $\left.-i t_{0}, i t_{0}\right]$ whose length is determined by the module of $R$.

Case II. $\operatorname{Re} \alpha>0$. We have to consider the differential equation

$$
w^{\prime}(t)^{2} \frac{w^{2}-w\left(1+|\alpha|^{2}\right) /(\operatorname{Re} \alpha)+1}{w(\alpha-w)(\bar{\alpha}-w)(1-\alpha w)(1-\bar{\alpha} w)}>0 .
$$

The numerator has the roots $w=x_{1}$ and $w=x_{2}$, where $0<x_{1} \leqq \operatorname{Re} \alpha$ and $x_{2}>1$. Clearly, $w(t)=x_{1} t(0<t<1)$ is a solution curve, and $\Gamma_{f}$ is either an interval $\left[0, t_{0}\right]$ (in particular, this happens if $\alpha>0$ and thus $z>0$ ), or $\Gamma_{f}$ consists of the interval $\left[0, x_{1}\right]$ and a symmetric fork at $x_{1}$.

Case III. $\operatorname{Re} \alpha<0$. The numerator again has two roots, one of which is $x_{3}\left(\operatorname{Re} \alpha \leqq x_{3}<0\right)$. The curve $w(t)=x_{3} t(0<t<1)$ is a solution, and the quadratic differential has a simple zero at $w=x_{3}$. The continuum $\Gamma_{f}$ is as in Case II, but reflected in the imaginary axis.

The following proposition now follows from the geometry of slit mappings.

Proposition 1. Let $z=\mathrm{re}^{i \theta}\left(r_{0}<r<1\right)$. The extremal value

$$
\max _{0 \leqq \theta<2 \pi} \max _{f \in \mathscr{F}_{s}}|f(z)|
$$

is attained if $z$ is real and $\Gamma_{f}$ is a horizontal line segment containing the origin as an endpoint and pointing toward $z$.

Suppose $f$ is an extremal function for the minimum problem

$$
\min _{g \in \mathscr{F}_{s}}|g(z)|
$$

We have to consider the differential equation $w^{\prime}(t)^{2} s(w(t))<0$, where $s(w)$ is given by (4).

Case I. $\operatorname{Re} \alpha=0$. The solution curve containing the origin is the real line, and $\Gamma_{f}$ is some interval $\left[-t_{1}, t_{2}\right]\left(t_{1} \geqq 0, t_{2} \geqq 0\right)$.

Case II. $\operatorname{Re} \alpha>0$. It is easy to verify that $w(t)=t(t<0)$ solves the differential equation.

Case III. $\operatorname{Re} \alpha<0$. In this case, $w(t)=t(t>0)$ is the solution containing the origin.

The following result is now obvious.

Proposition 2. Let $z=\operatorname{re}^{i \theta}\left(r_{0}<r<1\right)$. The extremal value

$$
\min _{0 \leqq \theta<2 \pi} \min _{f \in \mathscr{F}_{s}}|f(z)|
$$

is attained if $z$ is real and $\Gamma_{f}$ is a horizontal line segment containing the origin as an endpoint and pointing away from $z$.

Propositions 1 and 2 agree with the results found with other methods; see, for example, F. HuCKEMANN [7, Theorem 1]. 


\section{Distortion}

We now consider the distortion at a point $z \in R$, as measured by the absolute value of the derivative at $z$.

Suppose $f$ is an extremal function for the problem

$$
\max _{\mathbf{g} \in \mathscr{F}_{s}}\left|g^{\prime}(z)\right| \text {. }
$$

Set $\alpha=f(z)$. Then $\left|V_{\rho}^{s}(f(z))^{\prime}\right|=\left|\left(V_{\rho}^{s}\right)^{\prime}(\alpha) f^{\prime}(z)\right| \leqq\left|f^{\prime}(z)\right|$, which leads to the inequality $\operatorname{Re}\left\{a \rho^{2} s(w)+O\left(\rho^{3}\right)\right\} \leqq 0$, where

and

$$
s(w)=\frac{P(w)}{w(\alpha-w)^{2}(\bar{\alpha}-w)^{2}(1-\alpha w)^{2}(1-\bar{\alpha} w)^{2}}
$$

with

$$
P(w)=a_{0}+a_{1} w+a_{2} w^{2}+a_{3} w^{3}+a_{2} w^{4}+a_{1} w^{5}+a_{0} w^{6}
$$

$$
\begin{aligned}
& a_{0}=-2|\alpha|^{4} \operatorname{Re} \alpha, \\
& a_{1}=4|\alpha|^{4}+4|\alpha|^{6}+\left((\operatorname{Re} \alpha)^{2}-(\operatorname{Im} \alpha)^{2}\right)\left(-1+4|\alpha|^{2}+|\alpha|^{4}\right), \\
& a_{2}=\operatorname{Re} \alpha\left(2-8|\alpha|^{2}-18|\alpha|^{4}-4|\alpha|^{6}\right)-2|\alpha|^{2} \operatorname{Re} \alpha\left((\operatorname{Re} \alpha)^{2}-3(\operatorname{Im} \alpha)^{2}\right), \\
& a_{3}=-1+16|\alpha|^{4}+8|\alpha|^{6}+|\alpha|^{8}+\left((\operatorname{Re} \alpha)^{2}-(\operatorname{Im} \alpha)^{2}\right)\left(8|\alpha|^{2}+8|\alpha|^{4}\right) .
\end{aligned}
$$

The differential equation for $\Gamma_{f}$ is $w^{\prime}(t)^{2} s(w(t))>0$.

Case I. $\operatorname{Re} \alpha=0$. The differential equation for $\Gamma_{f}$ becomes

$$
w^{\prime}(t)^{2} \frac{1+w^{2}\left(-1+8|\alpha|^{4}+|\alpha|^{8}\right) /\left(|\alpha|^{2}+3|\alpha|^{6}\right)+w^{4}}{\left(|\alpha|^{2}+w^{2}\right)^{2}\left(1+|\alpha|^{2} w^{2}\right)^{2}}>0 \text {. }
$$

If $|\alpha|^{2} \geqq V / 5-2$, the numerator has no real root in $|w|<1$, and $\Gamma_{f}$ is an interval $\left[-t_{1}, t_{2}\right]\left(t_{1} \geqq 0, t_{2} \geqq 0\right)$. If $|\alpha|^{2}<\sqrt{5}-2$, let $x_{1}$ denote the smallest positive root of the numerator $\left(x_{1}\right.$ is a simple zero, and $x_{1} \rightarrow 0$ as $\left.|\alpha| \rightarrow 0\right)$. Then $\Gamma_{f}$ is a subset of the curve consisting of the interval $\left[-x_{1}, x_{1}\right]$ and sprouting symmetric forks at $x_{1}$ and $-x_{1}$.

Case II. $\operatorname{Re} \alpha>0$. If $w$ is small and negative, then $P(w)$ is negative. Choose $x_{2}\left(-1 \leqq x_{2}<0\right)$ such that $P(w)<0$ for $x_{2}<w<0$. Then, clearly, $w(t)=-t$ $\left(0<t<\left|x_{2}\right|\right)$ is a solution, and $\Gamma_{f}$ is either a slit $\left[-t_{0}, 0\right]$ or a slit $\left[x_{2}, 0\right]$ with a fork at $x_{2}$.

In particular, if $\alpha$ is positive, the polynomial $P(w)$ becomes

$$
P(w)=(w-\alpha)^{2}(1-\alpha w)^{2}\left[-2 \alpha^{3} w^{2}+\left(-1+4 \alpha^{2}+\alpha^{4}\right) w-2 \alpha^{3}\right],
$$

and the differential equation for $\Gamma_{f}$ simplifies to

$$
w^{\prime}(t)^{2} \frac{2 \alpha^{3} w^{2}+w\left(1-4 \alpha^{2}-\alpha^{4}\right)+2 \alpha^{3}}{w(w-\alpha)^{2}(1-\alpha w)^{2}}<0 .
$$

This is the same differential equation that DUREN [1, Section 2] obtained for this problem, and hence the solution is the same.

Case III. $\operatorname{Re} \alpha<0$. Solution curves for this case are reflections in the imaginary axis of solution curves for Case II. 
We have shown that the maximal distortion at a point $z \in R$ is obtained if $z$ is real. The omitted continuum $\Gamma_{f}$ is a horizontal slit from 0 away from $z$ (if $z$ is sufficiently large), or $\Gamma_{f}$ starts as such a slit and then sprouts a symmetric fork.

For the minimum problem

$$
\min _{g \in \mathscr{F}_{s}}\left|g^{\prime}(z)\right|,
$$

the differential equation for $\Gamma_{f}$, the continuum omitted by the extremal function $f$, becomes $w^{\prime}(t)^{2} s(w(t))<0$, where $s(w)$ is given by (5).

Case $I . \operatorname{Re} \alpha=0$. Computations show that $\Gamma_{f}$ is an interval, symmetric about the origin, of the imaginary axis.

Case II. $\operatorname{Re} \alpha>0$. Let $x_{1}$ denote the smallest positive root of $P(w)$ in $(0,1]$; if there is no such root, set $x_{1}=1$. Then $\Gamma_{f}$ is an interval $\left[0, t_{0}\right]$ if $t_{0} \leqq x_{1}$, or $\Gamma_{f}$ starts as the slit $\left[0, x_{1}\right]$ and then sprouts a fork at $x_{1}$. In particular, if $\alpha$ is positive, $\Gamma_{f}$ is a slit $\left[0, t_{0}\right]$.

Case III. $\operatorname{Re} \alpha<0$. Again, solutions for this case are reflections in the imaginary axis of solutions for Case II.

It follows that the minimal distortion at a point $z \in R$ is obtained if $z$ is real. The omitted continuum $\Gamma_{f}$ is a horizontal slit from 0 toward $z$.

\section{A Rotation Problem}

We consider the problem

$$
\max _{g \in \mathscr{F}_{s}} \operatorname{Im} \log \frac{g(z)}{z}
$$

(select the branch of the $\operatorname{logarithm}$ for which $\operatorname{Im} \log \frac{g(z)}{z}=0$ for $z=1$ ). Suppose that $f$ is an extremal function, and set $\alpha=f(z)$. Note that if $z$ is real, then $f(z)$ is real. Therefore, the problem is only meaningful if $z$ is not real, and consequently $\operatorname{Im} f(z)=\operatorname{Im} \alpha \neq 0$.

The inequality

leads to the relation

$$
\operatorname{Im} \log \frac{V_{\rho}^{s}(f(z))}{z} \leqq \operatorname{Im} \log \frac{f(z)}{z}
$$

$$
\operatorname{Re}\left\{a \rho^{2}\left[\frac{-i\left(1-\alpha^{2}\right)}{w_{0}\left(\alpha-w_{0}\right)\left(1-\alpha w_{0}\right)}+\frac{i\left(1-\bar{\alpha}^{2}\right)}{w_{0}\left(\bar{\alpha}-w_{0}\right)\left(1-\bar{\alpha} w_{0}\right)}\right]+O\left(\rho^{3}\right)\right\} \leqq 0 .
$$

Thus the omitted continuum $\Gamma_{f}$ satisfies the differential equation $w^{\prime}(t)^{2} s(w(t))<0$, where

$$
s(w)=(\operatorname{Im} \alpha) \frac{w^{2}-w(4 \operatorname{Re} \alpha) /\left(1+|\alpha|^{2}\right)+1}{w(\alpha-w)(\bar{\alpha}-w)(1-\alpha w)(1-\bar{\alpha} w)} .
$$

The numerator in (6) has no real roots, and it follows that

(i) if $\operatorname{Im} \alpha>0$, then $w(t)=-t(0<t<1)$ is a solution of the differential equation, and $\Gamma_{f}$ is a slit $[-c, 0]$ (the positive number $c$ depends on the module of $R$ ),

(ii) if $\operatorname{Im} \alpha<0$, then $w(t)=t(0<t<1)$ is a solution, and $\Gamma_{f}$ is a slit $[0, c]$. 
In striking contrast to the situation in the larger class $\mathscr{F}[9]$, we thus have the following result.

Proposition 3. Suppose $z$ lies in the upper half-plane. Then the maximum of the expression $\operatorname{Im} \log f(z) / z\left(f \in \mathscr{F}_{s}\right)$ is given by a unique slit map with slit $[-c, 0]$. (If $z$ lies in the lower half-plane, the slit is $[0, c]$.)

\section{Another Rotation Problem}

The rotation at a point can also be measured by the argument of the derivative. Choose the branch of the logarithm for which $\operatorname{Im} \log g^{\prime}(1)=0$, and consider the problem

$$
\max _{g \in \mathscr{F}_{s}} \operatorname{Im} \log g^{\prime}(z)
$$

(as in the previous section, this problem is meaningful only if $z$ is not real).

Suppose that $f$ is an extremal function, and set $\alpha=f(z)$. The variational method yields the differential equation $w^{\prime}(t)^{2} s(w(t))<0$ for the omitted continuum $\Gamma_{f}$, where

and

$$
s(w)=(\operatorname{Im} \alpha) \frac{Q(w)}{w(\alpha-w)^{2}(\bar{\alpha}-w)^{2}(1-\alpha w)^{2}(1-\bar{\alpha} w)^{2}}
$$

$$
Q(w)=a_{0}+a_{1} w+a_{2} w^{2}+a_{3} w^{3}+a_{2} w^{4}+a_{1} w^{5}+a_{0} w^{6},
$$

with

$$
\begin{aligned}
& a_{0}=|\alpha|^{4}, \\
& a_{1}=-(\operatorname{Re} \alpha)\left(|\alpha|^{4}+4|\alpha|^{2}+1\right), \\
& a_{2}=|\alpha|^{4}+4|\alpha|^{2}+1+\left(2+|\alpha|^{2}\right)\left(3(\operatorname{Re} \alpha)^{2}-(\operatorname{Im} \alpha)^{2}\right), \\
& a_{3}=-2(\operatorname{Re} \alpha)\left(4+4|\alpha|^{2}+(\operatorname{Re} \alpha)^{2}-(\operatorname{Im} \alpha)^{2}\right) .
\end{aligned}
$$

Case $I . \operatorname{Re} \alpha=0$. It is easy to check that solution curves for the differential equation are $w(t)=-t(0<t<1)$ if $\operatorname{Im} \alpha>0$, and $w(t)=t(0<t<1)$ if $\operatorname{Im} \alpha<0$.

Case II. $\operatorname{Re} \alpha>0$. If $\operatorname{Im} \alpha>0$, then $w(t)=-t(0<t<1)$ is a solution curve. If $\operatorname{Im} \alpha<0$, the curve may be more complicated. Let $x_{1}$ denote the smallest positive root of $Q(w)$ in the interval $(0,1)$, or set $x_{1}=1$ if there is no such root. (There may indeed be such an $x_{1}: Q(\operatorname{Re} \alpha)$ is negative for certain values of $\alpha$.) Then $w(t)=t\left(0<t<x_{1}\right)$ is a solution curve. At $x_{1}$, the curve sprouts a fork.

Case III. $\operatorname{Re} \alpha<0$. The solution curves are reflections in the imaginary axis of the solution curves for Case II.

We thus have the following result, which again differs considerably from the situation in the larger class $\mathscr{F}[9$, Section 4$]$.

Proposition 4. If $z$ lies in the upper half-plane, then the maximum of the expression $\operatorname{Im} \log f^{\prime}(z)\left(f \in \mathscr{F}_{s}\right)$ is given by a mapping for which the omitted continuum $\Gamma$ is either a slit $[-c, 0]$, where the positive number $c$ is determined by the module of $R$, or $\Gamma$ is a slit $\left[-x_{0}, 0\right]$ together with a symmetric fork at $-x_{0}$. The second 
case occurs only if $\operatorname{Re} f(z)<0$ and if $-c<-x_{0}$, where $-x_{0}$ denotes the smallest (in absolute value) negative root of $Q(w)$. (If $z$ lies in the lower half-plane, the omitted continua should be reflected in the imaginary axis.)

\section{A Convexity Question}

It is well-known that a normalized univalent function $f$ maps a circle $|z|=r$ onto a convex curve (that is, the interior of the curve is a convex region) if

$$
\operatorname{Re}\left(1+z \frac{f^{\prime \prime}(z)}{f^{\prime}(z)}\right)>0
$$

for $|z|=r$. We ask for the extremal values of the expression $\operatorname{Re}\left(1+z f^{\prime \prime}(z) / f^{\prime}(z)\right)$. To simplify the expressions, we assume that $z=r>0$.

Suppose $f$ is an extremal function for the problem

$$
\max _{g \in \mathscr{F}_{s}} \operatorname{Re}\left(1+r \frac{g^{\prime \prime}(r)}{g^{\prime}(r)}\right) \text {. }
$$

Comparing $f$ with its neighbors $V_{p}^{s} \circ f$, we find the differential equation $w^{\prime}(t)^{2} s(w(t))>0$ for the omitted continuum $\Gamma_{f}$, where

$$
s(w)=\frac{-\alpha^{3}+\left(3 \alpha^{2}+1\right) w-6 \alpha w^{2}+\left(3 \alpha^{2}+1\right) w^{3}-\alpha^{3} w^{4}}{w(\alpha-w)^{3}(1-\alpha w)^{3}} .
$$

Our assumptions imply that $\alpha=f(r)>0$. Hence $\Gamma_{f}$ is a slit $[-c, 0]$.

Suppose $f$ is an extremal function for the minimum problem

$$
\min _{g \in \mathscr{F}_{s}} \operatorname{Re}\left(1+r \frac{g^{\prime \prime}(r)}{g^{\prime}(r)}\right) \text {. }
$$

We find the differential equation $w^{\prime}(t)^{2} s(w(t))<0$, where $s(w)$ is given by (7). The numerator of $s(w)$ has exactly one positive real root inside the unit disk, which is given by the expression

$$
\begin{aligned}
x_{0}= & \frac{1}{4 \alpha^{3}}\left\{3 \alpha^{2}+1+\left(1-\alpha^{2}\right) \sqrt{8 \alpha^{2}+1}\right. \\
& \left.-\left[2\left(1-\alpha^{2}\right)\left(4 \alpha^{4}+7 \alpha^{2}+1+\left(3 \alpha^{2}+1\right) \sqrt{8 \alpha^{2}+1}\right)\right]^{\frac{1}{2}}\right\}
\end{aligned}
$$

(note that $0<x_{0}<\alpha$ ). Thus the omitted continuum $\Gamma_{f}$ is a slit $[0, c]$ (if $\alpha$ is large with respect to the module of $R$ ), or $\Gamma_{f}$ is the slit $\left[0, x_{0}\right]$ together with a symmetric fork at $x_{0}$.

Proposition 5. Fix $r\left(r_{0}<r<1\right)$. Then the maximum of the expression

$$
\operatorname{Re}\left(1+r \frac{f^{\prime \prime}(r)}{f^{\prime}(r)}\right) \quad\left(f \in \mathscr{F}_{s}\right)
$$

is given by a slit mapping, with slit from 0 away from $r$. The minimum is given by a slit mapping, with slit from 0 toward $r$, if $r$ is sufficiently large, or by a mapping whose omitted continuum is a slit $\left[0, x_{0}\right]$ together with a symmetric fork at $x_{0}$. 


\section{Curvature}

The curvature of the image of a circle $|z|=r$ under the function $f$ at $f(r)$ is given by the expression

$$
\frac{1}{\left|r f^{\prime}(r)\right|} \operatorname{Re}\left(1+r \frac{f^{\prime \prime}(r)}{f^{\prime}(r)}\right)
$$

We ask which functions in $\mathscr{F}_{s}$ maximize and minimize (8).

Suppose $f$ maximizes (8). Using our variational method, we find that $\Gamma_{f}$ satisfies the differential equation $w^{\prime}(t)^{2} s(w(t))>0$, where

and

$$
s(w)=\frac{a_{0}+a_{1} w+a_{2} w^{2}+a_{1} w^{3}+a_{0} w^{4}}{w(\alpha-w)^{3}(1-\alpha w)^{3}}
$$

$$
\begin{aligned}
& a_{0}=-2 \beta \alpha^{4}-2 \gamma \alpha^{3}, \\
& a_{1}=2 \gamma\left(3 \alpha^{2}+1\right)+\beta \alpha\left(3 \alpha^{4}+6 \alpha^{2}-1\right), \\
& a_{2}=-12 \alpha \gamma-\beta\left(\alpha^{6}+9 \alpha^{4}+3 \alpha^{2}-1\right), \\
& \alpha=f(r), \quad \beta=\operatorname{Re}\left(1+r f^{\prime \prime}(r) / f^{\prime}(r)\right), \quad \gamma=r f^{\prime}(r) .
\end{aligned}
$$

The numerator of $s(w)$ has a negative real root inside the unit disk if and only if

$$
\frac{1}{\gamma} \beta(1+\alpha)\left(\alpha^{2}+2 \alpha-1\right)<-4 \text {. }
$$

Thus the continuum $\Gamma_{f}$ is either a slit $[-c, 0]$ or a slit $\left[-x_{0}, 0\right]$ together with a symmetric fork at $-x_{0}$ (here $-x_{0}\left(\left|x_{0}\right|<1\right)$ denotes the smallest negative root of the numerator of $s(w))$.

If $f$ minimizes (8), then $\Gamma_{f}$ satisfies the differential equation $w^{\prime}(t)^{2} s(w(t))<0$, where $s(w)$ is as above. For each $\alpha(0<\alpha<1)$, the numerator of $s(w)$ has a root $x_{1}$ in the interval $(0, \alpha)$. Thus $\Gamma_{f}$ is a slit $[0, c]$ if $\alpha$ is large with respect to the module of $R$ (that is, if $c \leqq x_{1}$ ), or $\Gamma_{f}$ is a slit $\left[0, x_{1}\right]$ together with a symmetric fork at $x_{1}$.

The following proposition summarizes these results.

Proposition 6. The curvature of the image of the circle $|z|=r$ at the point $\alpha=f(r)$ is maximized by a slit mapping with slit from 0 away from $r$ (or, if $\alpha$ is sufficiently small and the module of $R$ is sufficiently large, by a map whose omitted continuum starts as a slit from 0 away from $r$ and then sprouts a symmetric fork). The curvature is minimized by a slit mapping with slit from 0 toward $r$ (or, if $\alpha$ is sufficiently small and the module of $R$ is sufficiently large, by a map whose omitted continuum starts as a slit from 0 toward $r$ and then sprouts a symmetric fork).

\section{A Distance Problem}

In this section, we ask which functions in $\mathscr{F}_{s}$ separate two symmetric points in $R$ the furthest and which functions move them the closest together. 
Fix $z \in R, \operatorname{Im} z \neq 0$. Suppose $f$ is an extremal function for the problem

$$
\max _{\mathbf{g} \in \mathscr{F}_{s}}|g(z)-g(\bar{z})|,
$$

and set $\alpha=f(z)$. The variational method gives the differential equation $w^{\prime}(t)^{2} s(w(t))<0$ for the omitted continuum $\Gamma_{f}$, where

$$
s(w)=\frac{2|\alpha|^{2} \operatorname{Re} \alpha-w\left(|\alpha|^{4}+4(\operatorname{Re} \alpha)^{2}-1\right)+w^{2} 2|\alpha|^{2} \operatorname{Re} \alpha}{w(\alpha-w)(\bar{\alpha}-w)(1-\alpha w)(1-\bar{\alpha} w)} .
$$

Case I. $\operatorname{Re} \alpha=0$. It is easy to check that $w(t)=i|\alpha| t(-1<t<1)$ solves the differential equation; hence $\Gamma_{f}$ is a vertical slit $\left[-i t_{0}, i t_{0}\right]$.

Case II. $\operatorname{Re} \alpha>0$. If $\operatorname{Re} \alpha \geqq\left(1-|\alpha|^{2}\right) / 2$, the numerator of $s(w)$ is positive for $-1<w \leqq 0$, and $\Gamma_{f}$ is a slit $[-c, 0]$. If $0<\operatorname{Re} \alpha<\left(1-|\alpha|^{2}\right) / 2$, then the numerator of $s(w)$ has a real root $-x_{0}\left(-1<-x_{0}<0\right)$, which tends to 0 as $\operatorname{Re} \alpha$ decreases to 0 . In this case, $\Gamma_{f}$ is a slit $[-c, 0]$ if the module of $R$ is large with respect to $\operatorname{Re} \alpha$, or $\Gamma_{f}$ is a slit $\left[-x_{0}, 0\right]$ together with a symmetric fork at $-x_{0}$.

Case III. $\operatorname{Re} \alpha<0$. If $\operatorname{Re} \alpha \leqq\left(|\alpha|^{2}-1\right) / 2$, then $\Gamma_{f}$ is a slit $[0, c]$. If $\left(|\alpha|^{2}-1\right) / 2<$ $\operatorname{Re} \alpha<0$ and if the module of $R$ is sufficiently large, then $\Gamma_{f}$ is a slit $\left[0, x_{1}\right]\left(x_{1}\right.$ denotes the smallest positive root of the numerator of $s(w))$ together with a symmetric fork at $x_{1}$.

Suppose $f$ is an extremal function for the minimum problem,

$$
\min _{g \in \mathscr{F}_{s}}|g(z)-g(\bar{z})|
$$

Then $\Gamma_{f}$ satisfies the differential equation $w^{\prime}(t)^{2} s(w(t))>0$, where $s(w)$ is as above. In this case, the zeros of $s(w)$ produce no branching of $\Gamma_{f}$. It is easy to check that $\Gamma_{f}$ is a horizontal slit containing the origin if $\operatorname{Re} \alpha=0, \Gamma_{f}$ is a slit $[0, c]$ if $\operatorname{Re} \alpha>0$, and $\Gamma_{f}$ is a slit $[-c, 0]$ if $\operatorname{Re} \alpha<0$.

We summarize:

Proposition 7. For fixed $z \in R(\operatorname{Im} z \neq 0)$, the minimum of the quantity

$$
|f(z)-f(\bar{z})| \quad\left(f \in \mathscr{F}_{s}\right)
$$

is attained by a slit mapping with slit along the real axis: the slit goes from 0 into the half-plane in which $f(z)$ lies, and if $\operatorname{Re} f(z)=0$, the slit contains 0 in its interior. The function maximizing (9) is as follows: $\Gamma_{f}$ is a vertical slit if $\operatorname{Re} f(z)=0 ; \Gamma_{f}$ is a slit $[-c, 0]$ if $\operatorname{Re} f(z)$ is sufficiently large and positive, or $\Gamma_{f}$ is a slit $\left[-x_{0}, 0\right]$ together with a symmetric fork at $-x_{0}\left(-x_{0} \rightarrow 0\right.$ as $\left.\operatorname{Re} f(z) \searrow 0\right) ; \Gamma_{f}$ is a slit $[0, c]$ if $\operatorname{Re} f(z)$ is sufficiently large and negative, or $\Gamma_{f}$ is a slit $\left[0, x_{1}\right]$ together with $a$ symmetric fork at $x_{1}\left(x_{1} \rightarrow 0\right.$ as $\left.\operatorname{Re} f(z) \nearrow 0\right)$.

\section{References}

1. DuREN, P. L., Distortion in certain conformal mappings of an annulus. Michigan Math. J. 10, 431-441 (1963).

2. Duren, P. L., \& M. SChIFFER, A variational method for functions schlicht in an annulus. Arch. Rational Mech. Anal. 9, 260-272 (1962). 
3. GAIER, D., Untersuchungen zur Durchführung der konformen Abbildung mehrfach zusammenhängender Gebiete. Arch. Rational Mech. Anal. 3, 149-178 (1959).

4. Gaier, D., \& F. HuCKEMANN, Extremal problems for functions schlicht in an annulus. Arch. Rational Mech. Anal. 9, 415-421 (1962).

5. Gehring, F. W., \& G. AF HÄLlströM, A distortion theorem for functions univalent in an annulus. Ann. Acad. Sci. Fenn. Ser. AI no. 325, 14 pp. (1963).

6. HuCKemanN, F., Ủber einige Extremalprobleme bei konformer Abbildung eines Kreisringes. Math. Z. 80, 200-208 (1962).

7. HuCKemanN, F., Extremal elements in certain classes of conformal mappings of an annulus. Acta Math. 118, 193-221 (1967).

8. McLaughlin, R., Extremalprobleme für eine Familie schlichter Funktionen. Math. Z. 118, 320-330 (1970).

9. McLaughlin, R., Some extremal problems for functions univalent in an annulus. Math. Scand. (to appear).

10. ScHIFFER, M., A method of variation within the family of simple functions. Proc. London Math. Soc. (2) 44, 432-449 (1938).

Department of Mathematics The University of Michigan-Flint

Flint, Michigan 48503

(Received July 20, 1971) 